sodium sulfate. After filtering the ether was evaporated and from the residue the pinakon was recovered as above. The yield was about $60 \mathrm{~g}$.

The dehydration of the pinakon was carried out according to the method mentioned by Thoerner and Zinke. ${ }^{1}$ One part of the pinakon is heated with three parts of acetic anhydride in a sealed tube for three hours at a temperature of $180^{\circ}$. The mixture is diluted with water, neutralized with sodium carbonate and subjected to a steam distillation. The hydrocarbon carried over with the steam readily crystallizes and is easily recrystallized from alcohol. Its melting point was found to be $49^{\circ}$. The readiness with which it spontaneously changes into a resinous material has already been mentioned, likewise the fact that satisfactory derivatives were not prepared.

SYRACUSE; N. Y.

[Contributions from the Chemical, Laboratory of Corneli University.]

\title{
DIPHENYLTETRACHLOROPHTHALIDE AND SOME OF ITS DERIVATIVES.
}

By W. R. ORndorfy and Miss R. R. MURtay.

Received December 26, 1916.

The tetrachlorophthalic acid used in this investigation was a commercial product purified by the method of Delbridge. ${ }^{2}$ The acid had a faint yellow

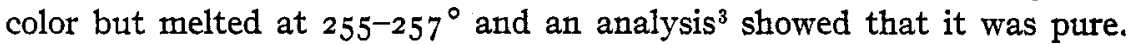

Subst., o.I 73I; cc. 0.I $N \mathrm{AgNO}_{2}, 22.19$.

Calc. for $\mathrm{C}_{8} \mathrm{H}_{2} \mathrm{O}_{4} \mathrm{Cl}_{4},{ }^{1} /{ }_{2} \mathrm{H}_{2} \mathrm{O}: \mathrm{Cl}, 45.33$. Found: $\mathrm{Cl}, 45.46$.

Tetrachlorophthalic anhydride was prepared from the acid by heating I 20 hours in a water oven at a temperature of about $100^{\circ}$, previous work ${ }^{4}$ having shown that this was sufficient time for its complete conversion into the anhydride.

Diphenyltetrachlorophthalide.-This substance was first prepared by the action of aluminum chloride on tetrachlorophthalyl chloride in benzene solution. The tetrachlorophthalyl chloride was prepared, with some changes, according to the method of Graebe. ${ }^{5}$ One molecule of the acid and 3.5 molecules of phosphorus pentachloride were heated in a flask connected with an upright condenser to $150-160^{\circ}$ on a metal bath for 30 hours. The phosphorus oxychloride was then distilled off and the residue, which did not pass over at $125^{\circ}$ under atmospheric pressure, was distilled under reduced pressure. A pale yellow liquid was obtained which immediately solidified to a pale yellow solid. This was recrystallized from benzene,

1 Loc. cit.

${ }^{2}$ Am. Chem. J., 4I, 4I4 (I909).

I Ibid., 4I, 397 (1909).

- Ibid., 4I, 359 (I909).

Ann., 238, 328 (1887). 
yielding a pure white crystalline chloride, melting sharply at $118^{\circ}$. The yield was $80 \%$ of the theoretical amount.

To prepare diphenyltetrachlorophthalide, $10 \mathrm{~g}$. of this chloride were dissolved in $100 \mathrm{cc}$. of benzene and $20 \mathrm{~g}$. commercial aluminum chloride in powdered form were added gradually to the solution. A very slight reaction took place in the cold, together with slight darkening in color of the mixture. Heated to the boiling point of benzene on a water bath, the reaction proceeded with foaming. Heating was continued for two hours, until no more hydrochloric acid gas came off. The reaction mixture was decomposed with water, distilled in steam to remove benzene, and the dark tarry solid residue, extracted with $10 \%$ sodium carbonate solution. This extract was found to contain only small amounts of 0 -benzoyltetrachlorobenzoic acid. The insoluble mass was washed with water and dried as completely as possible, then washed with cold ether. This removed almost all of the tar and left a light brown crystalline residue. After standing, the ether extracts yielded a few grams of crystalline substance which were added to the main mass and the whole recrystallized from glacial acetic acid. After three recrystallizations, the substance was obtained in short, thick needles, of a faint brown color, melting sharply at $250^{\circ}$. The yield was $80 \%$ of the theoretical amount. The product was analyzed for chlorine by the lime method described by Delbridge. ${ }^{1}$

Subst., I, 0.1640; II, 0.2130; cc. O.I $N \mathrm{AgNO}_{8}, \mathrm{I}, 15.48$; II, 20.07.

Calc. for $\mathrm{C}_{20} \mathrm{H}_{10} \mathrm{O}_{2} \mathrm{Cl}_{4}: \mathrm{Cl}, 33.45$. Found: $\mathrm{Cl}, \mathrm{I}, 33.47 ; \mathrm{II}, 33.4 \mathrm{I}$.

Diphenyltetrachlorophthalide is insoluble in alkalies and alkaline carbonates; almost insoluble in concentrated sulfuric acid, but on boiling is slightly soluble with a cherry-red color. It is precipitated from this solution in white flocks by addition of water. It is insoluble in methyl and ethyl alcohols, soluble in benzene on warming, slightly soluble in ether, and fairly soluble in glacial acetic acid. It is very soluble in acetone and chloroform. It may be recrystallized from benzene and alcohol, but best from glacial acetic acid. It resembles diphenylphthalide very closely in all its reactions.

Acetate of o-Benzoyltetrachlorobenzoic Acid.-o-Benzoyltetrachlorobenzoic acid was prepared by the method of Friedel and Crafts used by Kircher. ${ }^{2}$ One and five-tenths parts aluminum chloride were added gradually to a mixture of one part tetrachlorophthalic anhydride and 5 to 6 parts benzene. This was let stand half an hour and then warmed slowly to $50-70^{\circ}$ as long as hydrochloric acid came off, about one hour. Ten to fifteen grams aluminum chloride were then added, and the mixture warmed a quarter of an hour longer. The product being too viscous to pour, was removed to an evaporating dish, containing ice water. After

I Am. Chem. J., 4I, 397 (1909).

ann., 238, 338 (1887). 
decomposition was over, the mass was washed several times with hot water by decantation, and then dissolved in sodium carbonate solution, avoiding an excess. The sodium salt formed is easily recrystallized from hot water and, since a slight cooling will throw it out of its solution, this should be filtered hot using a hot water funnel. Four recrystallizations yielded a pure product, white and flaky in appearance. The salt was dissolved in water, steam passed in and hydrochloric acid added in excess until precipitation was complete. The acid was filtered off, washed till free from chlorides and dried. The product was colorless and finely crystalline. It melted at $200^{\circ}$, which is probably the melting point of the anhydride as the acetate (see below) melts at the same point.

The acetate was easily obtained by digesting one part of the acid with two parts acetic anhydride for two hours at the boiling point of the solution. Alcohol was added in equal volume and the solution allowed to cool, whereupon snow-white crystals separated in quantitative amount, melting at $200^{\circ}$. These were recrystallized from absolute alcohol, in which they are not very soluble, and yielded glistening white prisms melting at $200^{\circ}$. The acetate is insoluble in boiling aqueous alkali, but soluble in alcoholic alkali when heated, owing to saponification. It is slightly soluble in alcohol, benzene and toluene on heating. It contains no solvent of crystallization, but loses its acetyl group at $100^{\circ}$, as shown below. Air-dried crystals from absolute alcohol gave the following results:

Subst., I, 0.1525; II, 0.2094; cc. 0.I $N \mathrm{AgNO}_{3}, \mathrm{I}$, I4.98; II, 20.68 .

Calc. for $\mathrm{C}_{15} \mathrm{H}_{8} \mathrm{O}_{4} \mathrm{Cl}_{4}: \mathrm{Cl}, 34.94$. Found: $\mathrm{Cl}, \mathrm{I}, 34.83 ; \mathrm{II}, 35.02$.

The same crystals after heating for two hours at $96^{\circ}$ gave the following result:

Subst., 0.1496; cc. $0.1 \mathrm{~N} \mathrm{AgNO}_{3}, 15.38$.

Calc. for $\mathrm{C}_{10} \mathrm{H}_{8} \mathrm{O}_{4} \mathrm{Cl}_{4}: \mathrm{Cl}, 34.94$. Calc. for $\mathrm{C}_{14} \mathrm{H}_{6} \mathrm{O}_{3} \mathrm{Cl}_{4}: \mathrm{Cl}, 38.97$. Found: $\mathrm{Cl}, 36.46$. These figures show that some of the substance has lost an acetyl group and part of the acid or its anhydride has been regenerated, giving a high chlorine content. This accounts for the fact that the acetate melts at the same temperature as the acid itself. The acetate resembles von Pechmann's mixed anhydride of $o$-benzoylbenzoic acid and acetic acid very closely though it is a true acetate and not a mixed anhydride (p. 302).

Diphenyltetrachlorophthalide from the Acetate of o-Benzoyltetra-

chlorobenzoic Acid.-The acetate,

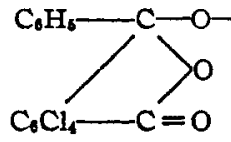

was condensed

with benzene by the Friedel and Crafts reaction according to von Pechmann's directions ${ }^{1}$ for making diphenylphthalide. Fifteen grams of the acetate were dissolved in $75 \mathrm{~g}$. benzene and aluminum chloride

1 Ber., 14, 1865 (1881). See also the theoretical part of this paper. 
added until no more hydrochloric acid gas was evolved. About $20 \mathrm{~g}$. were sufficient. The reaction proceeded vigorously in the cold and the solution became dark red and somewhat viscous. It was decomposed with water and distilled in steam to remove the excess of benzene. A pale yellow granular solid was obtained, uncontaminated with tar or other impurities. Two recrystallizations from glacial acetic acid gave a colorless, beautifully crystalline product, melting at $25^{\circ}$, identical in all its properties with the diphenyltetrachlorophthalide obtained above. Analyses gave the following results:

Subst., I, $0.2215 ;$ II, O.I 120; cc. $0.1 \mathrm{NAgNO}_{3}, \mathrm{I}, 20.87$; II, 10.56.

Calc. for $\mathrm{C}_{20} \mathrm{H}_{10} \mathrm{O}_{2} \mathrm{Cl}_{4}: \mathrm{Cl}, 33.45$. Found: $\mathrm{Cl}, 33.4 \mathrm{I}$; II, 33.43 .

The yield was quantitative and the crude product much purer than that prepared from tetrachlorophthalyl chloride. It is the best method for the preparation of diphenyltetrachlorophthalide.

Phenyltolyltetrachlorophthalide.-Five grams of the acetate of 0 benzoyltetrachlorobenzoic acid were dissolved in an excess of toluene by warming and Io $\mathrm{g}$. of aluminum chloride were gradually added in powdered form to the mixture. The reaction took place in the cold and the solution became dark reddish brown. The mixture was heated one-half hour on the water bath and decomposed with water. It was then distilled in steam. A pale yellow granular substance was obtained. After drying, it was recrystallized from glacial acetic acid giving shining pale yellow plates or needles, melting at $201^{\circ}$. After several recrystallizations the substance was obtained almost colorless. The melting point, however, did not change. The yield was quantitative. Analyses:

Subst., I, 0.1986; II, 0.I550; II, 0.196I; cc. 0.I $\mathrm{N} \mathrm{AgNO}_{3}$, I, I8.18; II, I4.09; III, I7.89. Calc. for $\mathrm{C}_{21} \mathrm{H}_{12} \mathrm{O}_{2} \mathrm{Cl}_{4}$ : Cl, 32.38. Found, $\mathrm{Cl}, \mathrm{I}, 32.46$; II, 32.23; III, 32.35.

The substance is insoluble in alcohol, soluble in benzene and can be crystallized from a mixture of these solvents but it is obtained in better form from glacial acetic acid. It closely resembles diphenyltetrachlorophthalide, of which it is a homolog.

Ditolyltetrachlorophthalide.-Five grams tetrachlorophthalyl chloride, $30 \mathrm{cc}$. toluene and Io $\mathrm{g}$. aluminum chloride were condensed by the same method used in case of diphenyltetrachlorophthalide. The reaction product after distilling in steam was similar to that obtained in the former experiment but was darker in color. It was extracted with 10\% sodium carbonate solution, the residue dried, washed with ether to remove tar and recrystallized from benzene and alcohol. A second recrystallization from glacial acetic acid gave shining scales or plates of a yellowish color. It was found impossible to remove this color even by boiling the solutions repeatedly with animal charcoal. This is similar to the result in case of the preparation of diphenyltetrachlorophthalide from the chloride. The latter, however, was obtained colorless by preparing it from 0 -benzoyl- 
tetrachlorobenzoic acid acetate. Ditolyitetrachlorophthalide melts at $209^{\circ}$. The yield was $80 \%$ of the theoretical amount. Analyses:

Subst., I, 0.2116; II, 0.1805; cc. 0.1 $N \mathrm{AgNO}_{3}$, I, I8.70; II, 15.99.

Calc. for $\mathrm{C}_{22} \mathrm{H}_{24} \mathrm{O}_{2} \mathrm{Cl}_{4}$ : Cl, 3I.38. Found: $\mathrm{Cl}, \mathrm{I}, 3 \mathrm{I} .34 ; \mathrm{II}, 3 \mathrm{I} .4 \mathrm{I}$.

Its solubilities are similar to those of diphenyltetrachlorophthalide and phenyltolyltetrachlorophthalide, with which substances it is holmologous.

Dinitrodiphenyltetrachlorophthalides. - The method of Baeyer used in the nitration of diphenylphthalide was followed. Ten grams diphenyltetrachlorophthalide were added slowly with shaking to roo g. nitric acid (sp. gr. I.52). The solution darkened in color as the solid dissolved and heat was evolved. The solution was allowed to stand overnight and then poured into ice water. A white curdy precipitate separated. This was filtered off and washed with water until free from acid. It was dried partially in the air and then in a water oven at $96^{\circ}$. The crude dry product melted at $120-140^{\circ}$ and weighed $\mathrm{I} 2 \mathrm{~g}$. (calculated $12.1 \mathrm{~g}$.). It was almost colorless and quite pure. It dissolved completely in methyl alcohol, from which it separated in small crystals having a pale yellow color, the first fraction melting higher than the second and third. Since the melting point was vague and the crystal form indefinite, it was judged to be a mixture. An analysis of the crystals which had stood in the air a long time was made.

Subst., 0.2142 ; cc. $0.1 \mathrm{~N} \mathrm{AgNO}_{3}$, 16.53.

Calc. for $\mathrm{C}_{20} \mathrm{H}_{8} \mathrm{O}_{6} \mathrm{Cl}_{4} \mathrm{~N}_{2}: \mathrm{Cl}, 27.59$. Found: $\mathrm{Cl}, 27.36$.

These figures and the indefinite melting point show that a mixture of dinitrodiphenyltetrachlorophthalides is present. The somewhat low value found for chlorine is due to the presence of some alcohol of crystallization (see below). The mixture was separated by fractional recrystallization from methyl alcohol, and from a mixture of benzene and methyl alcohol. It consists largely of the more soluble component. The less soluble member was finally obtained in small amount, about $0.8 \mathrm{~g}$. in ro. It separates from a saturated solution benzene on addition of methyl alcohol in glistening snow white small plates or leaflets melting at 238 $240^{\circ}$. It can be obtained from methyl alcohol alone in the form of a white powder having the same melting point. It is fairly soluble in benzene, difficultly in methyl and ethyl alcohols and gives colorless solutions in these solvents. It is very soluble in acetone and acetic acid; insoluble in ether, water and alkalies. It crystallizes from a mixture of benzene and methyl alcohol with two molecules of methyl alcohol of crystallization as shown by the following analysis of the air-dried product and determination of the loss in weight:

Subst., 0.1354; cc. 0.1 $N \mathrm{AgNO}_{3}$, 9.32.

Calc. for $\mathrm{C}_{20} \mathrm{H}_{3} \mathrm{O}_{6} \mathrm{Cl}_{4} \mathrm{~N}_{2,2} \mathrm{CH}_{4} \mathrm{O}: \mathrm{Cl}$, 24.54. Found: $\mathrm{Cl}, 24.41$.

A weighed portion of the substance was heated at $130^{\circ}$ to constant weight. 
Subst., (air-dried) 0.1726 ; loss in wt. at $130^{\circ}$, 0.0196 .

Calc. for $\mathrm{C}_{20} \mathrm{H}_{8} \mathrm{O}_{6} \mathrm{Cl}_{4} \mathrm{~N}_{2} .2 \mathrm{CH}_{4} \mathrm{O}: \mathrm{CH}_{3} \mathrm{OH}, \mathrm{I}$ I.08. Found: $\mathrm{CH}_{8} \mathrm{OH}$, ir.36. An analysis for chlorine was made on the crystals after heating to $130^{\circ}$. Subst., o.1530; cc. 0.1 $\mathrm{N} \mathrm{AgNO}_{3}, x$ x.89.

Calc. for $\mathrm{C}_{20} \mathrm{H}_{8} \mathrm{O}_{6} \mathrm{Cl}_{4} \mathrm{~N}_{2}: \mathrm{Cl}, 27.59$. Found: $\mathrm{Cl}, 27.56$.

The substance when dried lost its crystalline form and became opaque, powdery and slightly yellow in color. Nothing condensed on the sides of the tube on heating, showing that no water was present.

The more soluble component, of which the mixture mainly consisted, was obtained pure by the same method. It is much more soluble in methyl alcohol than its isomer and somewhat more soluble in benzene. The other solubilities are similar to those of the isomeric substance. It crystallizes from methyl alcohol in pale yellow feathery crystals, melting at I62$\mathrm{I} 64^{\circ}$, and from methyl aclohol alone in more finely divided form of the same melting point. It dissolves in alcohol forming a bright yellow solution. The crystals contain no solvent of crystallization. Air-dried crystals were analyzed for chlorine.

Subst., I, 0.2260; II, o.1850; cc. o.I $N \mathrm{AgNO}_{3}, \mathrm{I}, 17.53$; II, 14.36.

Calc. for $\mathrm{C}_{20} \mathrm{H}_{8} \mathrm{O}_{6} \mathrm{Cl}_{4} \mathrm{~N}_{2}: \mathrm{Cl}, 27.59$. Found: $\mathrm{Cl}, \mathrm{I}, 27.51 ; \mathrm{II}, 27.52$.

Traces of a third dinitro product were found but could not be obtained in large enough quantity to be purified and analyzed. It crystallized from a mixture of alcohol and benzene in cubes melting at $180-185^{\circ}$.

Diaminodiphenyltetrachlorophthalides. - An attempt was made to prepare the reduction products of the dinitrodiphenyltetrachlorophthalides described above. This was done by means of stannous chloride in acid solution. Ten grams of the nitro compounds were dissolved in $60 \mathrm{cc}$. glacial acetic acid and $15 \mathrm{~g}$. stannous chloride (calculated I3.I $_{\text {g.) }}$ ) were added to the warm solution. Twenty-five $\mathrm{cc}$. concentrated aqueous hydrochloric acid were then added little by little with shaking, so as not to precipitate the nitro compounds. The solution darkened from a clear yellow to a deep brownish red. After all the acid had been added, dilution to several hundred $\mathrm{cc}$. volume produced no precipitation, so that reduction was judged to be complete. The tin was removed as completely as possible by means of gaseous hydrogen sulfide. The solution was then made alkaline with sodium carbonate, whereupon a white amorphous precipitate separated. This was filtered off and dried. Extraction with alcohol left no residue. The extract on concentration yielded yellow crystals that did not melt at $300^{\circ}$.

Since on ignition a large residue remained the substance was judged to be a tin salt. Attempts were made to remove the tin by heating with concentrated hydrochloric acid. The substance dissolved with difficulty in the acid, separating out on cooling in fine needles. These were insoluble in cold water but soluble in hot water to some extent. They were de- 
composed with alkali, yielding a white amorphous precipitate similar to that first obtained. 'It left an ash on ignition and melted at $210-230^{\circ}$. The product (needles) from concentrated hydrochloric acid was probably the hydrochloride of the tin compound, since tin was not removed by this process, although repeated three times.

Hydroxydiphenyltetrachlorophthalide.-This substance was first prepared by Mr. H. C. Allen in this laboratory. After many attempts, mainly unsuccessful, to condense $o$-benzoyltetrachlorobenzoic acid with phenol with concentrated sulfuric acid according to Baeyer's ${ }^{1}$ directions for making hydroxydiphenylphthalide, tin tetrachloride; was employed. The following method was used: Io g. of o-benzoyl tetrachlorobenzoic acid were melted with Io g. phenol. Fifteen grams of tin tetrachloride were then added and the whole boiled gently on a metal bath at $150^{\circ}$ for three hours. The mixture became dark and tarry but on washing with water almost all of the dark material was removed and the residue was pinkish and granular. Ten grams of crude product were obtained. This, after thorough drying, was dissolved in ether and extracted with sodium carbonate. Practically nothing was shaken out. After evaporating off part of the ether, ligroin was added, which precipitated part of the substance. On standing the remainder came out. The whole was crystallized several times from glacial acetic acid, which yielded well-formed, colorless prisms, melting at $268-270^{\circ}$. The substance is slightly soluble in alkalies in the cold without color, but dissolves on heating with a decided yellow color. It is soluble in ordinary solvents but insoluble in water and ligroin. Air-dried crystals, on analysis for chlorine, gave the following figures:

Subst., I, 0.34I 7; II, 0.2840; cc. 0.I $N \mathrm{AgNO}_{3}, \mathrm{I}, 30.85$; II, 25.74 .

Calc. for $\mathrm{C}_{20} \mathrm{H}_{10} \mathrm{Cl}_{4} \mathrm{O}_{3}: \mathrm{Cl}, 32.23$. Found: $\mathrm{Cl}, \mathrm{I}, 32.01 ; \mathrm{II}, 32 . \mathrm{I} 4$.

Hydroxydiphenyltetrachlorophthalide was also prepared by heating the acetate of $o$-benzoyltetrachlorobenzoic acid with an excess of phenol on a metal bath to a temperature of $180-190^{\circ}$ for three hours. The viscous reaction product was distilled in steam to remove the excess of phenol and after drying dissolved in ether and precipitated with ligroin. This product was crystallized three times from glacial acetic acid which gave crystals that melted at $269-271^{\circ}$. It was colorless and identical in all respects with the product prepared by Mr. Allen. The yield was $90 \%$ of the theoretical amount. No condensing agent is necessary since acetic acid, not water, is evolved. Analysis of the air-dried product gave the following figures:

Subst., 0.2015 ; cc. $0.1 N \mathrm{AgNO}_{3}, 18.3 \mathrm{I}$.

Calc. for $\mathrm{C}_{20} \mathrm{H}_{10} \mathrm{Cl}_{4} \mathrm{O}_{3}: \mathrm{Cl}, 32.23$. Found: $\mathrm{Cl}, 32.22$.

The Acetate of Hydroxydiphenyltetrachlorophthalide.-This com${ }^{1}$ Ann., 354, I 7 ( 1907). 
pound was also prepared by Mr. H. C. Allen. One part of the hydroxydiphenylphthalide was boiled with one part sodium acetate and five parts acetic anhydride for one hour and the product precipitated with water. It was colorless and melted at $2 \mathrm{II}^{\circ}$ when pure. The air-dried crystals were analyzed for chlorine.

Subst., I, 0.2219 ; II, $0.22 I_{4}$; cc. $0.1 \mathrm{NAgNO}_{3}, \mathrm{I}, 18.34$; II, 18.30.

Calc. for $\mathrm{C}_{22} \mathrm{H}_{12} \mathrm{Cl}_{4} \mathrm{O}_{4}: \mathrm{Cl}, 29.42$. Found: $\mathrm{Cl}, \mathrm{I}, 29.3 \mathrm{I} ; \mathrm{II}, 29.3 \mathrm{I}$.

Hydroxydiphenyltetrachlorophthalide acetate is insoluble in methyl and ethyl alcohol, slightly soluble in acetone, crystallizing from it in plates. It is soluble in benzene and in glacial acetic acid.

Phenylresorcinoltetrachlorophthalein.-This substance was prepared by condensing $o$-benzoyltetrachlorobenzoic acid acetate and resorcinol. Three grams of the acetate and $1.2 \mathrm{~g}$. of resorcinol (calculated $0.8 \mathrm{I} \mathrm{g}$.) were heated on a metal bath to a temperature of $160^{\circ}$ for two hours. At the end of this time acetic acid ceased to come off and the reaction was judged to be complete. The contents of the flask were washed with hot water to remove the excess of resorcinol and after drying dissolved in ether. Ligroin was added and the solution allowed to stand, whereupon yellow crystal crusts separated. These were crystallized from $95 \%$ alcohol several times, a white crystal powder being obtained, that melted at $283^{-285^{\circ}}$, the substance darkening above $250^{\circ}$. These crystals appear to contain both alcohol and water of crystallization from the following analysis of the air-dried product:

Subst., 0.15I I; cc. o.I $N \mathrm{AgNO}_{8}$, I 1.69 .

Calc. for $\mathrm{C}_{20} \mathrm{H}_{10} \mathrm{O}_{4} \mathrm{Cl}_{4} \cdot \mathrm{C}_{2} \mathrm{H}_{6} \mathrm{O} \cdot \mathrm{H}_{2} \mathrm{O}: \mathrm{Cl}, 27.27$. Found: $\mathrm{Cl}, 27.43$.

A portion of the substance was dried in a tube heated to $120^{\circ}$ by electricity. ${ }^{1}$ Dry air was drawn through the tube and the vapors passed through a $20 \%$ solution of sodium carbonate containing iodine. Yellow crystals of iodoform separated after warming and then cooling the solution. As the drying proceeded, water condensed on the sides of the tube.

Subst., I, 0.1363; II, O.I8Io; loss at $125^{\circ}, \mathrm{I}$, 0.0153; II, 0.0215.

Calc. for $\mathrm{C}_{20} \mathrm{H}_{10} \mathrm{O}_{4} \mathrm{Cl}_{4} \cdot \mathrm{C}_{2} \mathrm{C}_{6} \mathrm{O} \cdot \mathrm{H}_{2} \mathrm{O}: \mathrm{C}_{2} \mathrm{H}_{6} \mathrm{O}$ and $\mathrm{H}_{2} \mathrm{O}$, 12.32. Found: $\mathrm{C}_{2} \mathrm{H}_{8} \mathrm{O}$ and $\mathrm{H}_{2} \mathrm{O}$, I, II.23; II, I I.88.

Analyses were made on the dry samples prepared as above:

Subst., I, 0.12 10; II, 0.1595; cc. 0.I $N \mathrm{AgNO}_{3}$, I, I0.6 I ; II, I 3.96.

Calc. for $\mathrm{C}_{20} \mathrm{H}_{10} \mathrm{O}_{4} \mathrm{Cl}_{4}$ : $\mathrm{Cl}, 3$ I. IO. Found: $\mathrm{Cl}, \mathrm{I}, 3$ I.09; II, 3 I.04.

The phthalein crystallizes from benzene in transparent leaflets containing benzene of crystallization.

Subst., 0.1266 ; loss at $130^{\circ}$, 0.0180 .

Calc. for $\mathrm{C}_{20} \mathrm{H}_{10} \mathrm{O}_{4} \mathrm{Cl}_{4} \cdot \mathrm{C}_{6} \mathrm{H}_{8}: \mathrm{C}_{6} \mathrm{H}_{8}, 14.62$. Found: $\mathrm{C}_{6} \mathrm{H}_{8}, 14.22$.

This dried sample was analyzed and gave the following figures:

Subst., o. I086; cc. O.I $N$ AgNO, 9.53.

Calc, for $\mathrm{C}_{20} \mathrm{H}_{10} \mathrm{O}_{4} \mathrm{Cl}_{4}: \mathrm{Cl}, 3$ r.ro. Found: $\mathrm{Cl}, 3 \mathrm{r}$.12.

1 Am. Chem. J., 48, 477 (1912). 
The phthalein is soluble slowly in cold normal caustic soda with deep orange color and is precipitated by acids from this solution in colorless flocks. It is easily soluble in cold ether and acetone and absolute alcohol. It is not so easily soluble in $95 \%$ alcohol. It is rather easily soluble in benzene, insoluble in ligroin. A mixture of benzene and alcohol yields the best crystals.

Diacetate of Phenylresorcinoltetrachlorophthalein.-This substance was obtained by boiling one part of the phthalein with three parts acetic anhydride for a period of two or three hours. Absolute alcohol was added and the solution allowed to cool, whereupon a fine, white crystalline solid separated, which on recrystallization from a mixture of glacial acetic acid and alcohol formed silky white needles, melting at $235^{\circ}$. These, after drying in the air, were analyzed for chlorine.

Subst., I, 0.I660; II, 0.I63I; cc. O.I $N \mathrm{AgNO}_{3}$, I, I2.I9; II, 12.04 .

Calc. for $\mathrm{C}_{20} \mathrm{H}_{8} \mathrm{O}_{4} \mathrm{Cl}_{4}\left(\mathrm{COCH}_{3}\right)_{2}: \mathrm{Cl}$, 26.26. Found: $\mathrm{Cl}, \mathrm{I}, 26.04 ; \mathrm{II}, 26.18$.

The diacetate contained no solvent of crystallization. It was insoluble in aqueous alkali but was slowly hydrolyzed by alcoholic alkali. It is soluble in hot acetic acid and slightly soluble in hot absolute ethyl alcohol. It is soluble in other organic solvents.

\section{Theoretical.}

Diphenyltetrachlorophthalide is analogous to diphenylphthalide prepared by Baeyer ${ }^{1}$ by a similar method. It is, therefore, a derivative of tetrachlorophthalide, ${ }^{2}$ (I) is the mother substance of phenoltetrachlorophthalein (III) and should be represented by Formula II.

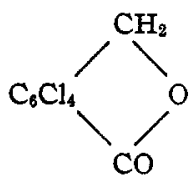

(I).

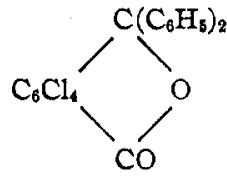

(II).

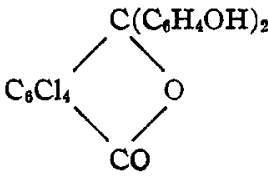

(III).

In view of the fact that the chief nitration product of diphenylphthalide can be converted into phenolphthalein, ${ }^{3}$ it is possible that the dinitrodiphenyltetrachlorophthalide obtained in largest amount and melting at $162-164^{\circ}$ is a dipara-nitration product of diphenyltetrachlorophthalide and that its isomer, melting at $238-240^{\circ}$ is the diortho compound. They are represented by the following formulas:
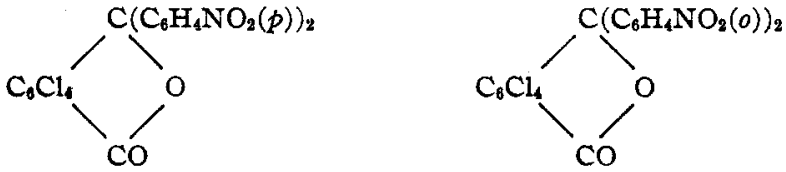

1 Ann., 202, 5Q (1880).

${ }^{2}$ Graebe, Ibid., 238, 330 (1887).

3 Ann., 202, 66 (1880). 
It is possible that the third unanalyzed compound is a dimeta-substitution product. The presence of four chlorine atoms in the molecule results in higher melting nitro products much more easily crystallized and separated than the substances prepared by Baeyer containing no chlorine. It is interesting to note that in the case of the dinitrodiphenyltetrachlorophthalides, the substance formed in largest amount is the low melting individual, not the high melting, as in the case of the dinitrodiphenylphthalides.

The substances phenyltolyltetrachlorophthalide and ditolyltetrachlorophthalide are homologs of diphenyltetrachlorophthalide and are probably para-compounds as shown below:

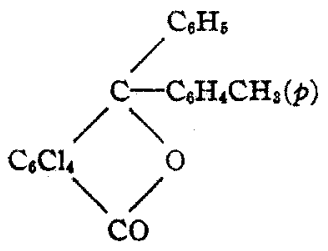

(I).

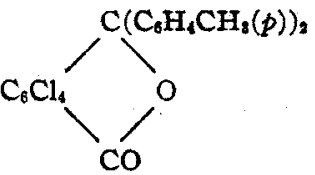

(II).

The methods by which they are prepared from 0 -benzoyltetrachlorobenzoic acid acetate and toluene and from tetrachlorophthalyl chloride and toluene are completely analogous to the two methods used in preparing the mothersubstance, diphenyltetrachlorophthalide.

Von Pechmann" prepared what he called the "mixed anhydride" of $o$-benzoylbenzoic acid and acetic acid and found that it reacted easily and quantitatively with benzene in the presence of aluminum chloride, giving a very pure diphenylphthalide. An analogous derivative of o-benzoyltetrachlorobenzoic acid has been prepared and this also possesses a great reactivity towards hydrocarbons in the presence of aluminum chloride and towards phenols without a condensing agent. This reactivity can be best explained by assuming that the compound has a lactone (I) rather than a ketone structure (II).

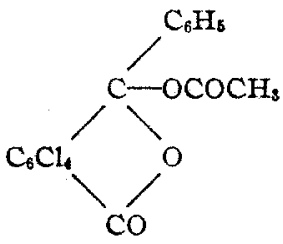

(I).<smiles>CCOC(=O)C(=O)OCC</smiles>

(II).

The reactivity is also increased by the fact that the acetyl group is lost at $100^{\circ}$, an anhydride of the acid resulting. The fact that the substance can be recrystallized from alcohol without the formation of an ester is

'Ber., I4, I865 (1881); see also Graebe, Ibid., 33, 2026 (I900). 
in agreement with Formula I for the acetate. A mixed anhydride (II) should give an ester. ${ }^{1}$

The constitution of the monohydroxydiphenyltetrachlorophthalide described in this paper is apparent from its preparation from the acetate of $o$-benzoyltetrachlorobenzoic acid and phenol. It is analogous to the monohydroxydiphenylphthalide prepared by von Pechmann ${ }^{2}$ and later studied by Baeyer. ${ }^{3}$
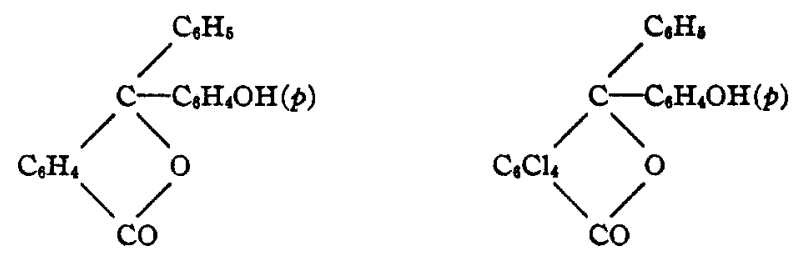

Monohydroxydiphenylphthalide. Monohydroxydiphenyltetrachlorophthalide.

Both substances yield monacetates and give bright yellow solutions with alkalies. They differ from phenolphthalein and phenoltetrachlorophthalein by one hydroxyl-group only and show the characteristic phthalein reaction towards alkalies and acids.

Phenylresorcinolphthalein and phenylresorcinoltetrachlorophthalein are isomers respectively of phenolphthalein and phenoltetrachlorophthalein.

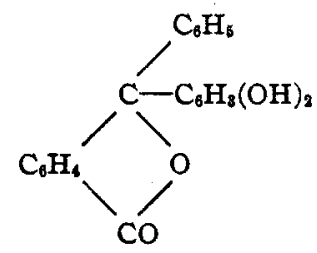

Phenylresorcinolphthalein.

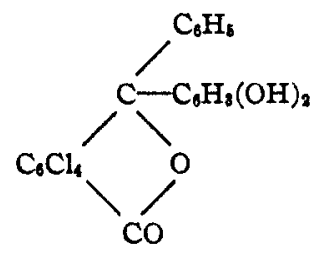

Phenylresorcinoltetrachlorophthalein.

The formation of a diacetate of phenylresorcinoltetrachlorophthalein proves the presence of two hydroxyl groups. Its solution in alkalies resembles that of monohydroxydiphenyltetrachlorophthalide. This shows that in order to obtain the red or violet salts, such as phenolphthalein and phenoltetrachlorophthalein give with alkalies, an hydroxyl group must be present in each benzene residue.

\section{Summary.}

I. Diphenyltetrachlorophthalide has been made from tetrachlorophthalyl cloride and also from the acetate of $o$-benzoyltetrachlorobenzoic acid. It resembles diphenylphthalide very closely in its properties and reactions.

2. Two dinitrodiphenyltetrachlorophthalides have been prepared by direct nitration of diphenyltetrachlorophthalide. The mixture of the

1 This Journal, 38, 2116 (I916).

2 Ber., 13, 1608 (1880).

${ }^{3}$ Ann., 354, I7) (1897). 
two isomeric dinitro-compounds has been separated and the components have been studied.

3. Attempts to prepare diaminodiphenyltetrachlorophthalides by reducing the dinitro-compounds with stannous chloride and hydrochloric acid did not give the desired product. Reduction took place, but the product contained tin, which could not be removed.

4. Phenyltolyltetrachlorophthalide and ditolyltetrachlorophthalide, homologs of diphenyltetrachlorophthalide, have been prepared.

5. Monohydroxydiphenyltetrachlorophthalide and phenylresorcinoltetrachlorophthalein have been prepared and have been found to give yellow and orange solutions, respectively, with alkalies. The acetates of these compounds have also been prepared.

6. The acetate of $o$-benzoyltetrachlorobenzoic acid has been prepared and found to possess the same reactivity towards hydrocarbons and phenols as von Pechmann's "mixed anhydride" of o-benzoylbenzoic acid and acetic acid.

ITHACA, N. Y.

[Contributron hrom Chemical Laboratory of Johns Hopkins University.]

STUDIES IN IDENTIFICATION. II. THE IDENTIFICATION OF PHENOLS.

BY E. EMMET REID.

Received December 6, 1916.

Introduction.

As is well known, the usual method for the identification of phenols is by making the benzoates by the Schotten-Baumann reaction. The reaction is not a convenient one to carry out and the yields are uncertain. Sometimes the benzoates prove to be liquids. It is convenient to have several methods of identification since one may apply in a certain case in which another gives poor results and, in important cases, it is desirable to prepare several crystalline derivatives so as to put the identification beyond a doubt.

In a previous paper ${ }^{1}$ it has been shown that $p$-nitrobenzyl bromide reacts readily with salts of many acids to form esters, many of which crystallize readily and have convenient melting points. It has also been found that this reagent reacts readily with the sodium or potassium phenolates to form $p$-nitrobenzyl ethers of the phenols and that these ethers frequently crystallize readily and have convenient melting points. The reaction with potassium phenolate is, of course,

$$
p-\mathrm{NO}_{2} \mathrm{C}_{6} \mathrm{H}_{4} \mathrm{CH}_{2} \mathrm{Br}+\mathrm{NaOC} \mathrm{H}_{6} \rightarrow p-\mathrm{NO}_{2} \mathrm{C}_{6} \mathrm{H}_{4} \mathrm{CH}_{2} \mathrm{OC}_{6} \mathrm{H}_{5}+\mathrm{NaBr} \text {. }
$$

One advantage of this method is that the derivatives obtained are ethers and are not, in general, so liable to be decomposed by saponifica-

1 This Journal, 39, I24 (1917). 